\title{
The End of Medicine as We Know It: Introduction to the New Journal, Systems Medicine
}

\author{
Jan Baumbach ${ }^{1,2}$ and Harald H.H.W. Schmidt ${ }^{3}$
}

Medicine has undoubtedly achieved a lot, often in collaboration with other sciences. Milestones include the first use of ether as an anesthetic by Crawford Long (1842), the germ theory by Louis Pasteur (1862) establishing that infection is caused by living organisms not by "spontaneous generation," the discovery of X-rays by Wilhelm Röntgen (1895), the discovery of penicillin in a mold by Alexander Fleming (1929), oral and intravenous rehydration solutions to treat infantile diarrhea by Daniel Darrow (1949), the link of tobacco and lung cancer (1950), the antibaby pill by Carl Djerassi (1951), the structure of DNA by Francis Crick and James Watson (1953), and the birth of evidence-based medicine by Archie Cochrane (1972).

One key area pertaining now to about $70 \%$ of a medical doctor's therapeutic approach, that is, prescribing a drug, is in a profound conceptual crisis: drug discovery and development. Importantly, this crisis reflects back on medicine in general and its remaining shortcomings despite all the mentioned achievements and milestones. The drug development crisis has not happened only recently as one might think, but drug discovery suffers from a conceptual problem that has caused a constant decline in efficacy since the 1950s. ${ }^{1}$ This has led to an exponential cost explosion without a gain in outcomes. Even worse, several drugs that were already on the market and part of therapeutic guidelines population-based studies failed to show patient-relevant benefits, and some drugs were later found to increase mortality. And most of the drugs that do work are often imprecise, as represented by high number of patients needed to treat over several years to achieve a benefit at least in one patient. ${ }^{2}$
The number one reason of drug development failures is a lack of efficacy. Part of this may be due to poor data quality and publication bias of the underlying basic biomedical science. ${ }^{3,4}$ A second reason is our current concept, definition and diagnosis of diseases. These are mostly defined by an apparent symptom (hypertension, asthma, and depression), affected organ (heart failure, retinopathy, and nephropathy), or the name of a doctor (Alzheimer and Parkinson). Drug therapy to a large extent relies on correcting these symptoms or normalizing risk factors (elevated blood pressure, cholesterol, and glucose). This is based on the assumption that by modulating surrogate parameters/risk factors, the relevant outcome (prevention of a heart attack, stroke, or death) will also be achieved. To some extent and for some of the treated patients, this is indeed the case, but for many this approach is of no benefit and they may be left with just the side effects of these drugs. Importantly, neither symptoms nor risk factors are mechanistic definitions of a disease. In fact, in most cases, we do not understand what exactly causes a disease. Noteworthy exceptions are rare diseases, where a precise (often single and severe) mutation can be diagnosed, thus a mechanism is known and sometimes a very specific therapy is available. If all diseases were mechanistically defined, it is predicted that even common diseases will segregate into less common or even rare diseases. To some degree, this has been achieved in oncology, where organ-based classifications have been replaced by cell-type or, in leukemia, mutation-specific definitions and treatments of signaling defects, although the overlap between cancer drivers and cancer drugs still leaves room for improvement. ${ }^{5}$

\footnotetext{
${ }^{1}$ Department of Experimental Bioinformatics, TUM School of Life Sciences Weihenstephan, Technical University of Munich, Munich, Germany.

${ }^{2}$ Computational Biology, Department of Mathematics and Computer Science, University of Southern Denmark, Odense, Denmark.

${ }^{3}$ Department of Pharmacology and Personalised Medicine, Cardiovascular Research Institute Maastricht (CARIM), Maastricht Centre for Systems Biology (MaCSBio), Maastricht University, Maastricht, the Netherlands.
}

(C) Jan Baumbach and Harald H.H.W. Schmidt 2018; Published by Mary Ann Liebert, Inc. This Open Access article is distributed under the terms of the Creative Commons License (http://creativecommons.org/licenses/by/4.0), which permits unrestricted use, distribution, and reproduction in any medium, provided the original work is properly cited. 
It is evident that the current medical taxonomy into mostly organ-based diseases and captured in the International Classification of Diseases in its current 10th version (ICD-10) is not sustainable. It assumes that diseases and pathomechanisms can be sufficiently defined and understood within one organ.

The advances in modern omics technology as well as their rapid and ongoing reduction in price have opened a whole new way of looking at diseases. Together with the drastic growth in public databases on molecular interactions, pathways, and gene regulations but also on comorbidities, drug (side)effects, etc., we have arrived at a new age that is emerging novel, integrative methods and approaches with clear potential to change future clinical decision-making. Novel big data analytics methodology will allow for in silico trials and a much higher degree of personalization in future medicine. We welcome all readers to this new journal, Systems Medicine, which is a novel research field emerging from systems biology and molecular medicine.

We are proud and very happy for the direct support of two very prominent researchers, who fortunately agreed to join us as senior associate editors:

1. Joseph Loscalzo, Harvard University, USA

2. Albert-László Barabási, Northeastern University, USA

In addition, we are extremely pleased to welcome the following individuals who will join us as associate editors:

1. Alvar Agusti, Hospital Clinic of Barcelona, Spain

2. Vasco Azevedo, Federal University of Minas Gerais, Brazil

3. Igor Jurisica, University of Toronto, Canada

4. Gordon Mills, MD Anderson Cancer Center, USA

5. Natasa Przulj, University College London, UK

6. Edwin Silverman, Harvard University, USA

We are glad that we have also been able to assemble a group of regional associate editors:

1. South America: Vasco Azevedo, Federal University of Minas Gerais, Brazil (also associate editor)

2. Japan: Hiroshi Mamitsuka, Kyoto University, Japan

3. Australasia: Tavpritesh Sethi, Indraprastha Institute of Information Technology, India

4. Africa: Nicholas Thomford, University of Cape Town, South Africa

5. China: Zefeng Wang, CAS-MPG Partner Institute for Computational Biology, China
And we are proud to say that we have been able to find enthusiastic support of 34 well-known international researchers, who agreed to form our initial editorial board.

In the coming years, you as authors together with us as a growing editorial team will jointly achieve a new understanding of disease, recognizing and appreciating comorbidities and endophenotypes using systems biology and network science tools, and validate and implement this into clinical practice. Publications in Systems Medicine will lead this process by bringing together interdisciplinary scientists and lowering the barriers for clinicians to appreciate big data science. Jointly, we will contribute to a new era of medicine, systems medicine.

We are excited to proceed with this new journal!

Our thanks go to the publisher, Mary Ann Liebert, Inc., who has made this dream real. The objectives of Systems Medicine are bold, necessary, and achievable.

\section{References}

1. Scannell JW, Blanckley A, Boldon $H$, et al. Diagnosing the decline in pharmaceutical R\&D efficiency. Nat Rev Drug Discov. 2012;11:191-200.

2. Schork NJ. Personalized medicine: time for one-person trials. Nature. 2015:520:609-611.

3. Kleinschnitz C, Mencl S, Kleikers PW, et al. NOS knockout or inhibition but not disrupting PSD-95-NOS interaction protect against ischemic brain damage. J Cereb Blood Flow Metab. 2016;36:1508-1512.

4. Kleikers PWM, Hooijmans C, Göb E, et al. A combined pre-clinical metaanalysis and randomized confirmatory trial approach to improve data validity for therapeutic target validation. Sci Rep. 2014;5:13428.

5. Santos R, Ursu O, Gaulton A, et al. A comprehensive map of molecular drug targets. Nat Rev Drug Discov. 2017;16:19-34.

Cite this article as: Baumbach J, Schmidt HHHW (2018) The end of medicine as we know it: Introduction to the new journal, Systems Medicine, Systems Medicine 1:1, 1-2, DOI: 10.1089/sysm.2017.28999.jba.
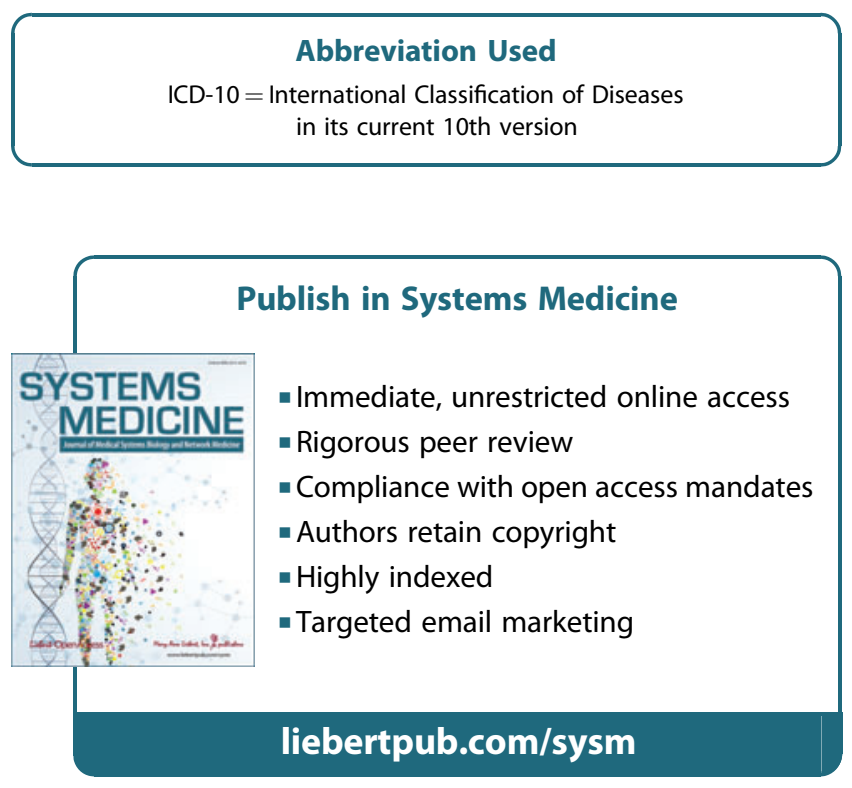